http://jmscr.igmpublication.org/home/ ISSN (e)-2347-176x ISSN (p) 2455-0450 crossref DOI: https://dx.doi.org/10.18535/jmscr/v9i3.11

\author{
(D) Journal Of Medical Science And Clinical Research \\ IGM Publication \\ An Official Publication of IGM Publication
}

\title{
Rhabdoid Meningioma of Brain - A Rare Tumor
}

\author{
Authors \\ Dr Jyothi Jonnadula ${ }^{1 *}$, Dr K Srinivas Rao ${ }^{1}$, Dr Anandh Balasubramaniam², \\ Dr Krishna Kumar ${ }^{2}$, Dr Milap Shah ${ }^{3}$ \\ ${ }^{1}$ *Department of Radiation Oncology, ${ }^{2}$ Department of Neurosurgery, ${ }^{3}$ Department of Pathology \\ Yashoda Hospital, Secunderabad, Telangana, India \\ *Corresponding Author \\ Dr Jyothi Jonnadula \\ Department of Radiation Oncology, Yashoda Hospital, Secunderabad, 500003, Telanagana, India
}

Abstract
Rhabdoid mengioma is a rare and aggressive tumor seen in young and middle aged group. It carries worst
prognosis with high recurrence rates and leptomeningeal dissemination. The longest survival observed
was 9 months with a median survival of 18 weeks. Here we report a case of Rhabdoid Meningioma of
brain in a 16 year old boy.
Keywords: Rhabdoid meningiona, Aggressive meningioma, Meningioma, Aggressive brain tumor.

Keywords: Rhabdoid meningiona, Aggressive meningioma, Meningioma, Aggressive brain tumor.

\section{Introduction}

Meningiomas are slow-growing tumors arising from the meningeal coverings of the brain and spinal cord. Although most meningiomas are benign, some distinct subtypes are associated with high recurrences, especially those composed histologically of rhabdoid cells. Rhabdoid meningioma is a rare and highly aggressive type with high recurrence rates and was classified as WHO grade III meningioma. It is derived from the arachnoidal epithelial cells and resembles rhabdomyoblasts, i.e., with abundant eosinophilic cytoplasm, eccentrically placed nuclei, and paranuclear inclusions that ultrastructurally correspond to whorled bundles of intermediate filaments.

Rhabdoid meningiomas account for $0.004 \%$ of all meningiomas. The recurrence rate is nearly $87 \%$ with a mortality of around $53 \%$. The presentation depends on the site of the lesion. Radiologically, it shows cystic and solid components with homogenous enhancement of the solid component associated with extensive peritumoral edema. Central necrosis and bone destruction are indicative of aggressive behaviour.

\section{Case-Report}

A 16 year old boy with history of vomiting, intermittent pulsatile sensation over left temple, neck pain, giddiness and weight loss of $10 \mathrm{~kg}$ in last two months was evaluated with MRI Brain which showed $5.3 \times 3.8 \times 4.7 \mathrm{~cm}$ lesion in left paramedian frontal lobe with mild perilesional edema, mass effect and midline shift (Figure 1). Postcontrast study reveals thick asymmetrical peripheral rim enhancement of the lesion, associated with nodular enhancement within posterior aspect of the lesion and adjacent leptomeningeal enhancement over the falx. He underwent left frontal craniotomy and excision of 
lesion on 15.12.2020. Postoperative histopathological examination showed Rhabdoid Meningioma,WHO Grade III with presence of necrosis along with vascular and brain invasion (Figure 2). On immunohistochemistry, tumour cells focally express EMA and S100 (Figure 3); GFAP, Desmin, Myogenin, HMB 45, LCA , MUM-1, PR, CK are negative; INI-1 shows retained nuclear expression; Mib-1 index is around $10 \%$. Later, he was advised CSF cytology and MRI spine screening to rule out leptomeningeal spread. Patient's general condition was deteriorated to undergo any further investigations and succumbed to death.
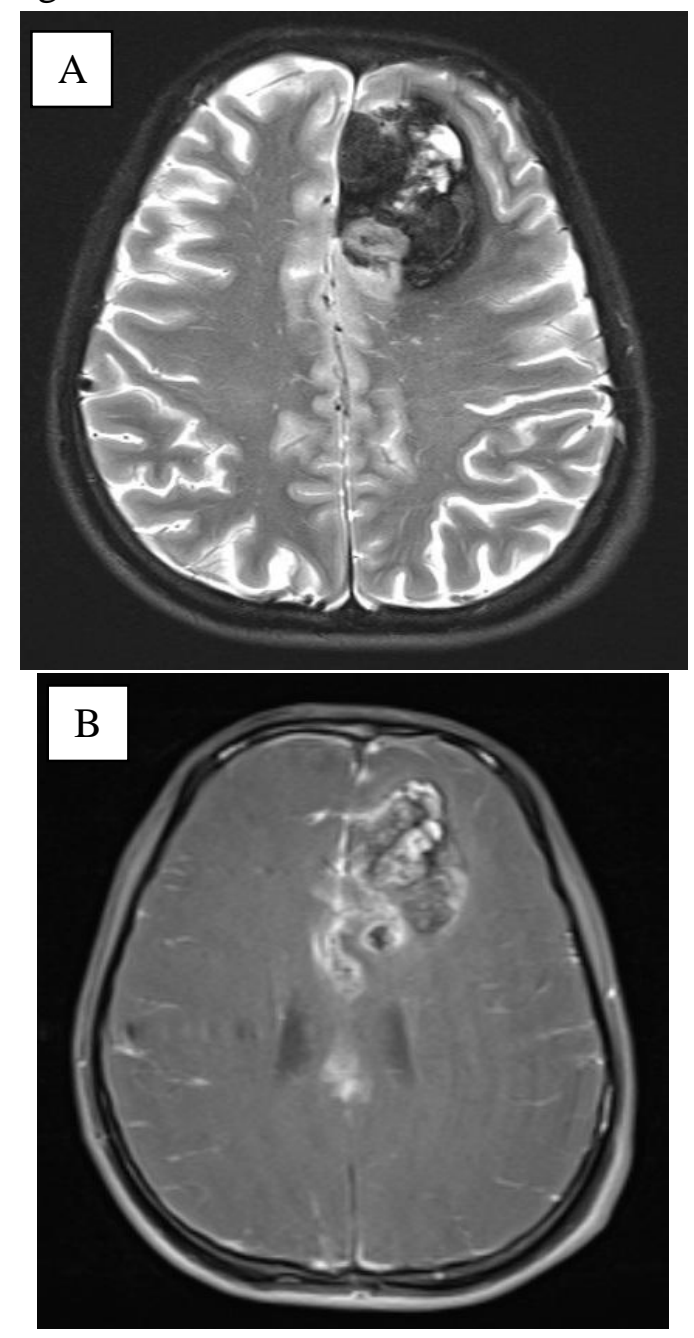

Figure 1

A) Large lobulated heterogeneous area of altered signal intensity measuring $53 \times 38 \times 47 \mathrm{~mm}$ noted in left paramedian frontal lobe involving cortex and subcortical white matter with mild perilesional oedema and mild mass-effect, with midline shift to right by $7 \mathrm{~mm}$. Lesion appears predominantly hypointense on $\mathrm{T} 2$ and FLAIR sequences with few hyperintense areas within.

B) Postcontrast study reveals thick asymmetrical peripheral rim enhancement of the lesion, associated with nodular enhancement within posterior aspect of the lesion.
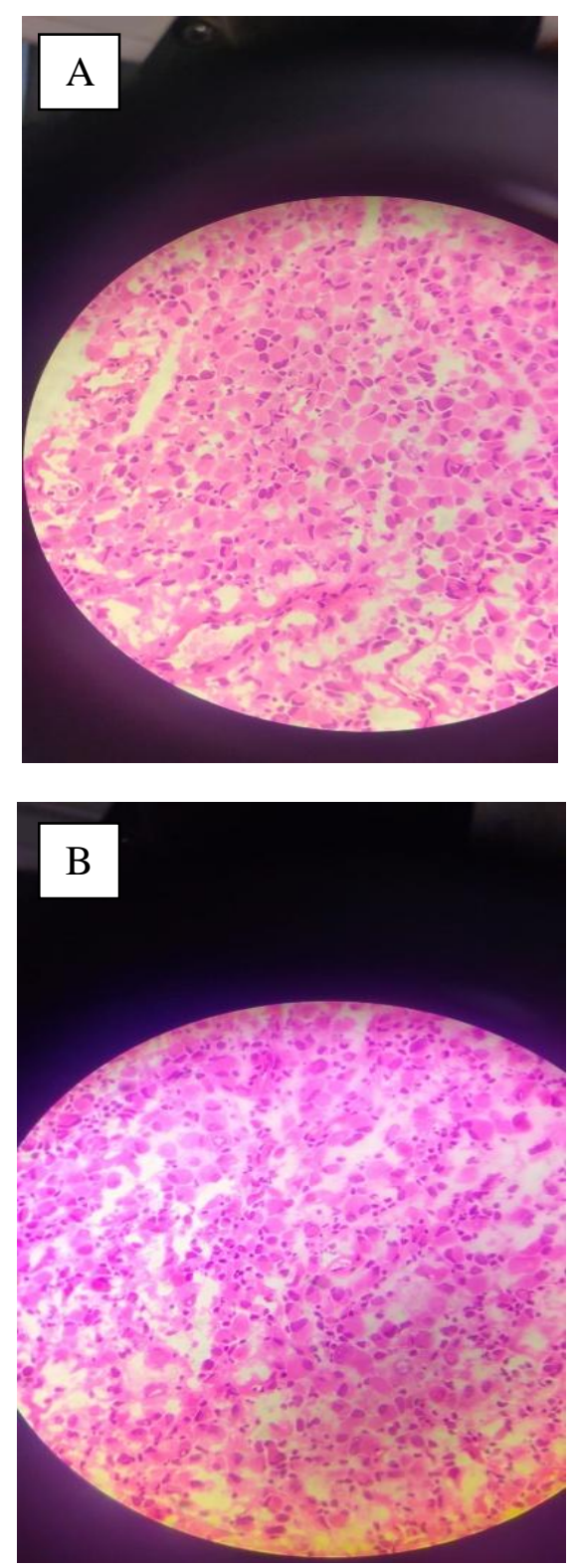

Figure 2

A) Tumor shows large rhabdoid cells with eccentrically placed nuclei and abundant dense eosiniphilic cytoplasm. Nuclei shows moderate atypia. Brain and vascular invasion was noted along with haemorrhage and necrosis.

B) Rhabdoid meningioma with intranuclear inclusions. 

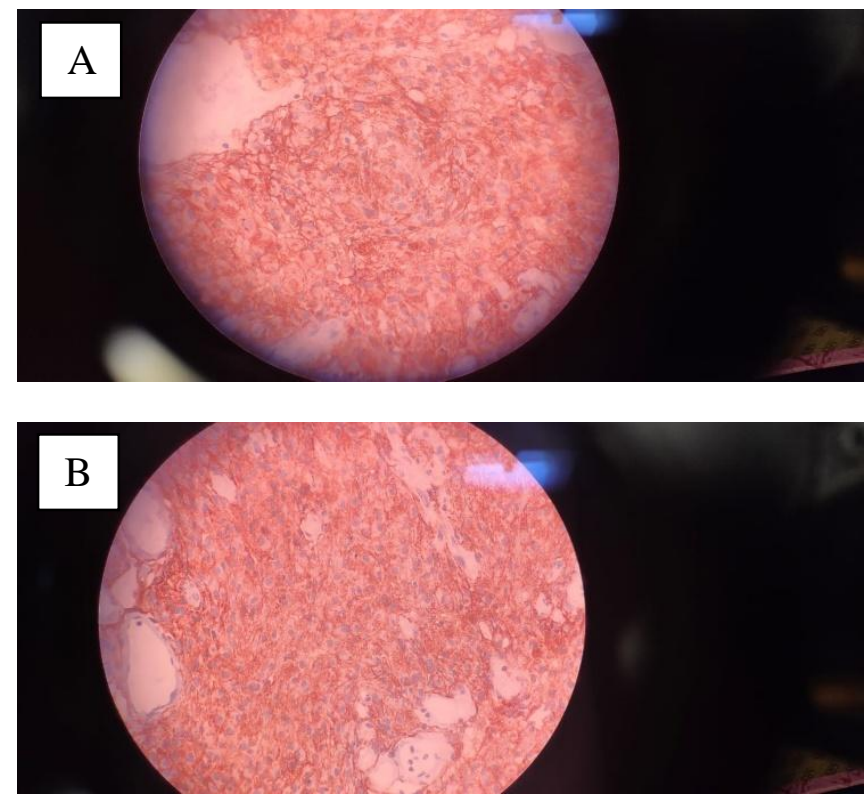

Figure 3

A) EMA positive B) S100 positive. Tumor cells are negative for LCA, CD 138, HMB 45, Myogenin, Desmin, GFAP. Tumor shows retained nuclear expression of INI1

\section{Discussion}

Rhabdoid meningioma is a rare and highly aggressive type of meningioma with high recurrence rates $^{(1)}$ and was included in WHO classification 2000 as a grade III subtype of meningioma $^{(2)}$. Rhabdoid refers to the resemblance of tumor to rhabdomyoblast, without true skeletal muscle differentiation ${ }^{(3)}$. Kepes et al and Perry et al first described the first two series of rhabdoid transformation of meningiomas in $1998^{(4)}$. It may be supratentorial, infratentorial or spinal. In our case, it was in supratentorial location. It is most commonly seen in young and middle aged group with equal rates of incidence in both males and females. Brain invasion, anaplasia, and extracranial metastasis have been reported. These meningiomas often recur, and the rhabdoid features become more apparent in subsequent biopsies. Recurrence may be due instead to metastases from the original tumour; along the subdural space, via CSF, drop metastases or haematogenous spread.

The cytologic features include abundant eosinophilic cytoplasm, eccentric nuclei, and intracytoplasmic hyaline inclusions. Diagnosis depends on finding evidence of meningothelial differentiation either by light microscopy (whorls, intranuclear pseudoinclusions), immunohistochemistry (expression of vimentin, epithelial membrane antigen, and progesteronereceptor positivity), or electron microscopy (interdigitating cytoplasmic membranes, intercellular junctions). Besides the rhabdoid morphology, cytoarchitectural features of atypical meningioma (four or more mitoses per 10 highpower fields, high cellularity, sheeting architecture, nuclear atypia, and necrosis) are seen in most cases. Expression of cytokeratin is common, but it is negative in our case.

The term malignant rhabdoid tumor (MRT) was first used to describe a distinctive pediatric renal tumor $^{(5)}$. The term was then extended to similar extrarenal pediatric tumors (extrarenal MRT), including atypical teratoid/rhabdoid tumor (AT/RT) of the central nervous system ${ }^{(6)}$. These tumors commonly show mutation of the INI1 gene on 22q11.2. In our case, IHC panel and morphology rules out differential diagnosis of atypical teratoid rhabdoid tumor, melanoma, plasmacytoma, metastatic carcinoma, rhabdomyosarcoma and lymphoma.

There is very limited data in the literature regarding the ideal management of rhabdoid meningioma due to rarity of the disease and its aggressive nature. They are notorious for the involvement of adjacent structures like important vessels and nerves. Hence, complete resection of the tumor becomes difficult and it requires aggressive treatment.

\section{References}

1. Kakar V, Elsmore A. Rhabdoid meningioma: Rare and highly aggressive. Internet J Neurosurg. 2005;3:1-5.

2. Matyja E, Grajkowska W, Nauman $P$, Bonicki W, Bojarski P, Marchel A. Necrotic rhabdoid meningiomas with aggressive clinical behavior. Clin Neuropathol. 2010;29:307-16.

3. Kar A, Rath G, Mohanty L, Behera P, 
Mohapatro S, Lenka A, et al. Rhabdoid meningioma-An uncommon and aggressive variant. $J$ Cancer Sci Ther. 2013;5:343-5.

4. Perry A, Scheithauer BW, Stafford SL, Abell-Aleff PC, Meyer FB. "Rhabdoid" meningioma: An aggressive variant. Am J Surg Pathol. 1998;22:1482-90.

5. Haas JE, Palmer NF, Weinberg AG, Beckwith JB, Hum Pathol. 1981 Jul; 12(7):646-57.

6. Rorke LB, Packer RJ, Biegel JA, J Neurosurg. 1996 Jul; 85(1):56-65. 